0245_IBEROS_1_E and CIBER-BBN for funding. CIBER-BBN is an initiative of the ISCIII.

Disclosure of Interests: Paula Casal-Beiroa: None declared, Vanesa Balboa-Barreiro: None declared, Noa Goyanes: None declared, Purificación Filgueira-Fernández : None declared, Pío González: None declared, Sonia Pertega: None declared, Francisco J. Blanco Consultant for: AbbVie, Bioiberica, BMS, GSK, Grünenthal, Janssen, Lilly, Pfizer, Regeneron, Roche, Sanofi, TRB Chemedica, and UCB, joana magalhães: None declared DOI: 10.1136/annrheumdis-2019-eular.5972

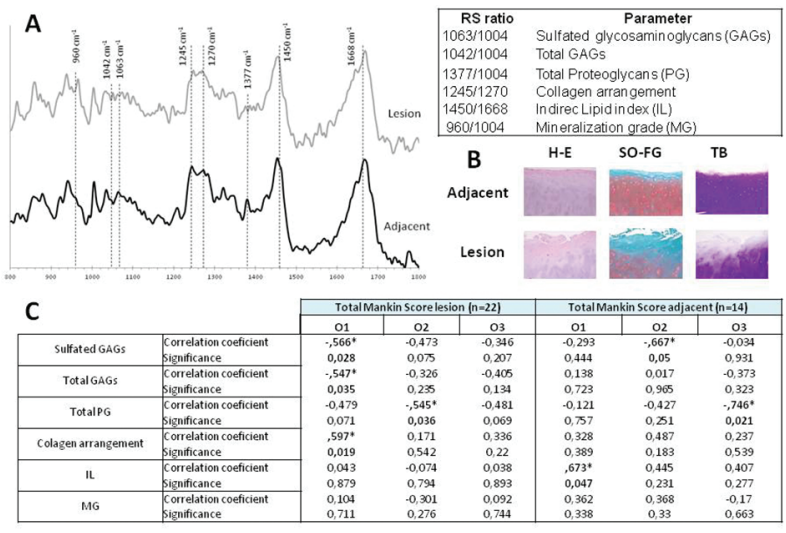
(Hematoxilin-eosin (H-E) Safranin-O/Fast-Green (SO-FG) and Toluidin Blue (TB)) used for MS analysis from the adjacent and lesion sites from a selected donor; : r rho Spearman's correlation coeficients and significance between RS and MS (total scoring) in lesion and adjacent sites for Observer 1, 2 and 3 ( 01,2 and 3 ).

\section{FRI0506 ROLE OF MITOCHONDRIA FROM PATIENTS WITH OA IN CELLULAR SENESCENCE}

Andrea Dalmao-Fernandez ${ }^{1}$, Tamara Hermida Gómez ${ }^{1}$, Maria Eugenia Vazquez Mosquera ${ }^{1}$, Sara Relaño-Fernandez ${ }^{1}$, Ignacio Rego-Perez ${ }^{1}$, Francisco J. Blanco ${ }^{1}$ Mercedes Fernandez-Moreno ${ }^{1,2} .{ }^{1}$ Instituto de Investigación Biomédica de $A$ Coruña (INIBIC), A coruña, Spain; ${ }^{2} C I B E R-B B M$, Madrid, Spain

Background: Chondrosenescence, chondroptosis and autophagy contribute to cell death and tissue damage in OA. The mitochondria are related with these three process implicated in the cartilage degeneration. Mitochondrial dysfunction is well documented in $\mathrm{OA}$ and has the capacity to promote abnormalities in chondrocyte viability contributing to cartilage degeneration. Cybrids are optimal cellular models to study the mitochondrial function, since they carry different mitochondrial variants with the same nuclear background, therefore, excluding the variations because of nuclear genome.

Objectives: The aim of this work is to study the role of human OA mitochondria in the cellular senescence, apoptosis and autophagy.

Methods: Cybrids were developed using 143B.TK Rho-0 cell line (nuclear donor) and platelets (mitochondrial donors) from healthy $(\mathrm{N})$ and knee OA donors. Senescence level was measured by real-time PCR method The percentage of mitochondrial depolarized was evaluated incubating cells with DilC1(5) in presence of FCCP $1 \mu \mathrm{M}$ using Flow Cytometer. The percentage of apoptotic cells was measured by Flow Cytometry using Annexin-V. Autophagy was evaluated through the developed of Microtubule-associated protein 1A/1B-light chain 3 (LC3) WB. Appropriate statistical analyses were performed with GraphPad Prism v6.

Results: The gene expression corresponding to senescence marker protein (SMP30) showed higher levels in OA cybrids than in N (4.535 \pm 1.63 ; $1.21 \pm 0.42$ respectively, $\mathrm{p} \leq 0.0005)$. OA cybrids showed higher increment depolarized mitochondria under negative stimuli in comparison with basal condition than $N(2.57 \pm 1.20 ; p \leq 0.0005 ; 1.76 \pm 0.99 ; p \leq 0.05$ respectively $)$. The analysis of apoptotic levels, when the cells were submitted for a positive stimuli with staurosporine $(2 \mu \mathrm{M})$ and an inflammatory environment with IL-1 $\beta(10 \mathrm{ng} / \mathrm{ml})$, OA cybrids reflected a statistically significant increase in positive cells for Anexine- $\mathrm{V}$ in comparison with $\mathrm{N}$ cybrids in both conditions (staurosporine $15.68 \pm 6.39 ; 6.41 \pm 4.88$ respectively, $p \leq 0.05$. IL-1 $0.924 \pm 0.19 ; 0.47 \pm 0.24$ respectively, $\mathrm{p} \leq 0.05$ ). Autophagy was analyzed studying LC3 a marker for autophagosome formation and the results showed that LC3 activation was reduced in OA cybrids (1.19 $\pm 0.24 ; 1.41 \pm 0.21$ respectively, $p \leq 0.05$ ).
Conclusion: Mitochondria from OA patients is involved in cellular senescence, apoptosis and autophagy (three relevant processes involved in $\mathrm{OA})$.

Disclosure of Interests: Andrea Dalmao-Fernandez: None declared, Tamara Hermida Gómez: None declared, Maria Eugenia Vazquez Mosquera: None declared, Sara Relaño-Fernandez: None declared, Ignacio RegoPerez: None declared, Francisco J. Blanco Consultant for: AbbVie, Bioiberica, BMS, GSK, Grünenthal, Janssen, Lilly, Pfizer, Regeneron, Roche, Sanofi, TRB Chemedica, and UCB, Mercedes Fernandez-Moreno: None declared

Doi: 10.1136/annrheumdis-2019-eular.3667

\section{$\mathrm{FR} 10507$ THE HUMAN-BASED IN VITRO 3D ARTHRITIC JOINT MODEL}

Alexandra Damerau, Annemarie Lang, Moritz Pfeiffenberger, Timo Gaber, Frank Buttgereit. Charité - Universitätsmedizin Berlin, corporate member of Freie Universität Berlin, Humboldt-Universität zu Berlin, and Berlin Institute of Health Department of Rheumatology and Clinical Immunology, Berlin, Germany

Background: Our ultimate goal is to develop a valid human in vitro 3D joint model to simulate the pathogenesis of arthritis. The model consists of different components including an osteogenic and chondrogenic part, the joint space with synovial fluid and the synovial membrane. It contains all involved cell types and thus, to allow interactions between cells by cell contacts and signaling molecules. The arthritic joint is simulated by the application of sorted immune cells and typical cytokines. As an alternative experimental setup for animal models, our in vitro 3D joint mode will enable us to study the influence and efficacy of potential drug candidates. Currently, there is no valid 3D model, which is able to mimic an arthritic joint.

Objectives: Here, we aimed to develop the single joint components, namely the osteogenic and chondrogenic part, the joint space with synovial fluid and the synovial membrane, separately and in co-culture without and with the application of neutrophils (PMNs) and cytokines.

Methods: We have used human bone marrow derived mesenchymal stromal cells (hMSCs) to develop the different 3D tissue components that are characterized in detail (e.g. cell vitality, structural integrity) using histological, biochemical and molecular biological methods as well as $\mu \mathrm{CT}$ and scanning electron microscope (SEM). For the osteogenic component, we populated $\beta$-tricalcium phosphate (TCP) - mimicking the mineral bony part - with hMSCs, while the scaffold-free 3D cartilage component was generated from a marrow tissue exclusively consisting of hMSC under intermittent mechanical stimulation (fzmb $\mathrm{GmbH}$ ). Simulating the synovia fluid, non-animal stabilized hyaluronic acid was applied to the osteochondral model. In order to model the synovial membrane, a confluent hMSC layer was formed on a polycarbonate membrane. Subsequently, we applied sorted $\mathrm{CD}_{15}{ }^{+}$cells to the synovial membrane and cytokines (e.g TNF, MIF, IL6) into the synovial fluid, analyzed using GPCR, multiplex immunoassay and flow cytometry.

Results: We developed an in vitro 3D bone model by successfully seeding hMSC on a $\beta$-TCP scaffold. Cells consistently adhere onto the scaffold for up to 6 weeks as observed by SEM. The analysis of cell viability via LDH detection and LIVE/DEAD staining showed no toxic effects on the cells even after 3 weeks of incubation as compared to the corresponding control. mRNA expression of bone-related genes such as RUNX2, SPP1 and COL1A1 as well as $\mu \mathrm{CT}$ analysis confirmed the osteogenic phenotype of hMSC grown in 3D. Mimicking the articular cartilage component, we verified its chondrogenic phenotype by $\mathrm{HE}$ and Alcian Blue staining as well as by the reduced mRNA expression of COL1A1 and an abundant expression of COL2A1. Interestingly, co-cultivation of the osteogenic and chondrogenic part looks promising showing a close alignment and cell interaction between both components. Modelling the synovial membrane, we successfully and reproducibly created a confluent hMSC cell layer, with a high cell viability even after 3 weeks of incubation. In addition, we injected cytokines into the synovial fluid. In response, the simulated synovial membrane showed an upregulation of IL6, HIF and MMP13 expression on mRNA level. Furthermore, under cocultural conditions the hMSCs enhanced the survival of the PMNs.

Conclusion: Results from the analysis of the single components confirmed viability, integrity and morphology pointing towards an ultimately successful development of the anticipated in vitro 3D model. By combining the different components in a standard 96 well format, we aim to provide a mid-throughput system for preclinical drug testing as well as a valid in vitro human-based 3D disease model to study the pathogenesis of arthritis. 